\title{
Novel mutations explain secondary resistance to imatinib
}

A possible mechanism of secondary resistance to imatinib in gastrointestinal stromal tumors (GIST) has been delineated in a new study. Chun-Meng Wang and colleagues identified novel KIT mutations in exon 13 or exon 17 in patients with GIST who acquired resistance to imatinib therapy.

The tyrosine kinase inhibitor imatinib, which targets the KIT receptor, illicits a favorable response in most patients with GIST. However, resistance to therapy eventually occurs with a median time to progression of 2 years.

Although KIT mutations have been implicated in a large proportion of GIST, the molecular response of KIT inhibition by imatinib is poorly understood. Thus, Wang et al. evaluated KIT mutations and downstream signaling profiles in 32 patients with GIST who had been treated with imatinib and surgical resection.

Activating mutations in KIT were observed in 26 patients. Furthermore, of the 14 patients who acquired secondary imatinib resistance, 11 had secondary KIT mutations, of which nine were in exon 17 and two in exon 13. The expression levels of KIT and AKT were higher in GIST with secondary KIT mutations and acquired imatinib resistance compared with imatinibresponsive GIST with primary KIT mutations.

In conclusion, the novel KIT mutations in exon 13 or exon 17 are involved in the molecular mechanism of secondary resistance to imatinib. Furthermore, the PI3K/AKT pathway might be important in these patients, notes Wang Chun Meng. "We should pay more attention to the signal transduction pathway in the imatinib-resistant GIST," he adds.

Lisa Richards

Original article Wang, C. M. et al. Molecular mechanisms of secondary imatinib resistance in patients with gastrointestinal stromal tumors. J. Cancer Res. Clin. Oncol. 136, 1065-1071 (2010) 